\title{
Phase diagram and superconductivity of calcium borohyrides at extreme pressures
}

\author{
Simone Di Cataldo, ${ }^{1,2, *}$ Wolfgang von der Linden, ${ }^{1}$ and Lilia Boeri ${ }^{2, \dagger}$ \\ ${ }^{1}$ Institute of Theoretical and Computational Physics, Graz University of Technology, NAWI Graz, 8010 Graz, Austria \\ ${ }^{2}$ Dipartimento di Fisica, Sapienza Università di Roma, 00185 Roma, Italy
}

(Received 1 June 2020; accepted 7 July 2020; published 29 July 2020)

\begin{abstract}
Motivated by the recent discovery of near-room temperature superconductivity in high-pressure superhydrides, we investigate from first principles the high-pressure superconducting phase diagram of the ternary Ca-B-H system, using $a b$ initio evolutionary crystal structure prediction, and Density Functional Perturbation Theory. We find that below $100 \mathrm{GPa}$ all stable and weakly metastable phases are insulating. This pressure marks the appearance of several new chemically-forbidden phases on the hull of stability, and the first onset of metalization in $\mathrm{CaBH}_{5}$. Metallization is then gradually achieved at higher pressure at different compositions. Among the metallic phases stable in the Megabar regime, we predict two high- $T_{\mathrm{c}}$ superconducting phases with $\mathrm{CaBH}_{6}$ and $\mathrm{Ca}_{2} \mathrm{~B}_{2} \mathrm{H}_{13}$ compositions, with critical temperatures of 119 and $89 \mathrm{~K}$ at $300 \mathrm{GPa}$, respectively, surviving to lower pressures. Ternary hydrides will most likely play a major role in superconductivity research in the coming years; our study suggests that, in order to reduce the pressure for the onset of metallicity and superconductivity, further explorations of ternary hydrides should focus on elements less electronegative than boron.
\end{abstract}

DOI: 10.1103/PhysRevB.102.014516

\section{INTRODUCTION}

The search for high-temperature superconductors in hydrogen-rich systems stems from two seminal intuitions of Neil Ashcroft's, i.e., that hydrogen, under sufficiently high pressures, may be turned into a metallic room-temperature superconductor, and that the same result may be achieved at lower pressures exploiting chemical precompression in hydrides [1,2]. This second intuition, in particular, led to a major breakthrough in 2014, with the experimental discovery that sulfur hydride $\left(\mathrm{SH}_{3}\right)$, under a pressure of $200 \mathrm{GPa}$, becomes a superconductor with a critical temperature $\left(T_{\mathrm{c}}\right)$ of $203 \mathrm{~K}[3,4]$. After the discovery of $\mathrm{SH}_{3}$, other superconducting hydrides were found, with lanthanum hydride $\left(\mathrm{LaH}_{10}\right)$ currently holding the record, with a $T_{\mathrm{c}}$ of $260 \mathrm{~K}$ [5-10]. All these high- $T_{\mathrm{c}}$ hydrides are remarkable examples of high-pressure forbidden chemistry, as their H-rich chemical compositions defy the standard rules on oxidation states and coordination; it is arguable that their existence would have never been anticipated, without theoretical predictions enabled by ab initio methods based on density functional theory (DFT) methods for crystal structure prediction and superconductivity, developed in the last 20 years [11-16].

High- $T_{\mathrm{c}}$ superhydrides belong to the class of conventional superconductors, where the superconducting pairing is mediated by phonons. For this class of materials, MigdalEliashberg theory, combined with first-principles calculations of the electron-phonon coupling, permits to make quantitative predictions of superconducting properties. Based on MigdalEliashberg theory and experience, the highest $T_{\mathrm{c}}$ among conventional superconductors are expected in materials, which

\footnotetext{
*simone.dicataldo@uniroma1.it

†lilia.boeri@uniroma1.it
}

(i) contain light elements, exhibit (ii) a sizable density of states (DOS) at the Fermi level, typical of good metals, and (iii) large electron-phonon $(e-p h)$ matrix elements, characteristic of systems with contain directional (covalent or ionic) bonds $[17,18]$. Whereas the first requirement is satisfied by any compound containing hydrogen, which is the lightest element in the periodic table, the other two conditions are met only by a few hydrides, and only at extreme pressures.

In the last five years, many research groups, galvanized by the ground-breaking $\mathrm{SH}_{3}$ discovery, devoted their efforts to the exploration of all possible binary hydrides, i.e., compounds with chemical formula $\mathrm{A}_{x} \mathrm{H}_{y}[16,19-22]$. As of 2020, this exploration has been essentially completed, showing that only around $10 \%$ of the elements of the periodic table form high-pressure binary hydrides with superconducting $T_{\mathrm{c}} \mathrm{s}$ exceeding nitrogen boiling temperature $(77 \mathrm{~K})$. These can be grouped essentially in two main families, depending on the size and electronegativity of the $A$ element.

The first family is that of covalent hydrides, in which $A$ can be a metalloid or nonmetal. At low pressure, covalent hydrides form molecular crystals, which are large-gap insulators [23-25]. Pressures in the megabar (100 GPa) range can render some of these compounds metallic, through band overlap. Some of these metallic molecular hydrides, such as phosphorous, silicon or sulfur hydrides, exhibit superconductivity, with $T_{c}$ s that can be as high as $100 \mathrm{~K}$ [9,26-33]. Increasing pressure even further, in a few systems molecular bonds break, and, as additional hydrogen is incorporated into the A-H lattice, compounds with completely different stoichiometries and geometrical motifs form, in which hydrogen and the guest atom arrange in highly symmetric structures, which are metallic and held together by covalent, directional bonds. This is the case, for example, of $\mathrm{SeH}_{3}$ and $\mathrm{SH}_{3}$, where $T_{c}$ 's can be as high as $200 \mathrm{~K}[3,4,34-37]$. 
The second family of high- $T_{\mathrm{c}}$ binary hydrides comprises weak hydrogen formers, such as alkaline earths, lanthanoids, actinoids, and early transition metals. Within this family, the highest $T_{\mathrm{c}} \mathrm{s}$ are usually found in hydrogen clathrate structures, characterized by highly symmetric $\mathrm{H}$ cages surrounding a central atom [38]. Several of these clathrate hydrides, which form at pressures around $100 \mathrm{GPa}$, were predicted to achieve superconducting $T_{c} s$ above $200 \mathrm{~K}[8,38-42]$, and $\mathrm{YH}_{6}, \mathrm{YH}_{9}$, $\mathrm{LaH}_{10}, \mathrm{ThH}_{10}$ were experimentally confirmed as near-room temperature superconductors $[5-8,10]$.

After the binary hydrides have been thoroughly explored, the natural route to improve the superconducting properties of high-pressure hydrides, either by reducing the pressures needed to achieve high $T_{\mathrm{c}}$, or by increasing the maximum $T_{\mathrm{c}}$, is to consider ternary hydrides-materials with $\mathrm{A}_{x} \mathrm{~B}_{y} \mathrm{H}_{z}$ composition, where the presence of two different elements, $A$ and $B$, opens many routes for the optimization of material properties. However, the increased flexibility of ternary hydrides comes at a high price, since accounting for possible decomposition paths requires exploring a much larger compositional space than in the case of binaries. For this reason, only a few out of the six-thousand possible ternary hydrides have been investigated to date [43]. Most studies in literature have been focusing on optimization of known hydrides, by iso- or eterovalent substitution [36,44-50], and only a few works took into account the full thermodynamics of ternary phases [51,52].

In this work, we used first-principles methods for evolutionary structure prediction [53] and superconductivity [12,54] to explore computationally the full high-pressure phase diagram of the calcium-boron-hydrogen (Ca-B-H) ternary system to identify new superconductors. Calcium boron hydrides are part of the broad family of metal-boronhydrides $(\mathrm{MBH})$, which have been extensively studied for hydrogen storage applications at room pressure. [55,56]. $\mathrm{MBH}$ are characterized by the simultaneous presence of a weak (M) hydride former, and a strong (B) hydride former, which forms strong (covalent or ionic) bonds with hydrogen. At ambient conditions, metal borohydrides adopt a $\mathrm{M}\left(\mathrm{BH}_{4}\right)_{n}$ stoichiometry, in which tetrahedral $\mathrm{BH}_{4}^{-}$anions, similar to methane, are loosely arranged on an open lattice, and accomodate mono-, di- or trivalent cations in the interstitials. Besides the stable $\mathrm{M}\left(\mathrm{BH}_{4}\right)_{n}$ compositions, other metastable phases have been predicted to form [57], containing $\mathrm{BH}_{3}^{-}$and $\mathrm{BH}_{2}^{-}$anions, with ethane- and polyethylene-like motifs. The similarity between $\mathrm{MBH}$ and hydrocarbons makes them very attractive candidates for superconductivity, since they combine in a single compound the strong directional bonds typical of covalent hydrides [24], with the possibility of controlling charge doping via interstitial atoms. Indeed, a theoretical study in lithium boron hydride showed that, while at ambient pressure all stable and metastable phases are wide-gap ionic insulators, at pressures in the megabar range covalent metallic structures with increased hydrogen content should become stable; a high- $T_{\mathrm{c}}$, metallic, highly symmetric $\mathrm{Li}_{2} \mathrm{BH}_{6}$ phase with a $T_{\mathrm{c}}$ of $98 \mathrm{~K}$ was predicted to form at $100 \mathrm{GPa}$ [51].

In this paper, we will analyze the high-pressure phase diagram of $\mathrm{Ca}-\mathrm{B}-\mathrm{H}$ with the precise aim of identifying new hydrogen-rich, covalent metallic structures, analogous to $\mathrm{SH}_{3}$, exhibiting high $-T_{\mathrm{c}}$ conventional superconductivity. We will show that, although such structures do indeed form, electronic structure features amenable to the chemical properties of calcium and boron limit the maximum $T_{\mathrm{c}}$ achievable and their stability range, making calcium borohydrides not competitive with the record binary hydrides, such as $\mathrm{SH}_{3}$ and $\mathrm{LaH}_{10}$.

The paper is organized as follows. In Sec. II, we will present the phase diagram as a function of pressure, highlighting the evolution of the structural properties of the stable phases. In Sec. III, we will present the electronic properties of the stable and metastable phases, focusing in more detail on the new metallic structures that we identify at high pressures. In Sec. IV, we will then investigate the electron-phonon properties, and discuss which specific electronic and structural features lead to low or high- $T_{\mathrm{c}}$ in the different phases. In Sec. V, we will draw the main conclusions of our work. The Appendix contains an extensive account of the computational details. Figures of the electronic DOS for all structures and band structure plots for selected high-pressure phases are reported in the Supplemental Material [58].

\section{PHASE DIAGRAM}

The Ca-B-H phase diagram at ambient pressure is wellcharacterized, since it was extensively studied for hydrogen storage applications. The ground state phase $\mathrm{Ca}\left(\mathrm{BH}_{4}\right)_{2}$ decomposes above $300{ }^{\circ} \mathrm{C}$ through different channels: either towards $\mathrm{CaH}_{2}, \mathrm{H}_{2}$, and $\mathrm{CaB}_{12} \mathrm{H}_{12}$, or towards $\mathrm{CaH}_{2}, \mathrm{CaB}_{6}$, and $\mathrm{H}_{2}$, through an intermediate $\mathrm{Ca}\left(\mathrm{BH}_{3}\right)_{2}$ phase [59-63]. These experimental observations are reproduced by firstprinciples calculations, which showed that the two pathways are quasidegenerate [57].

To the best of our knowledge, there are no predictions on the behavior of the ternary phase diagram under pressure, including intermediate $\mathrm{Ca}-\mathrm{B}-\mathrm{H}$ compositions. However, the pressure behavior of the binary systems (Ca-B, Ca-H, B-H), which form the edge of the ternary hull, was studied by $a b$-initio calculations in recent years, in search for possible superconductors. Superconducting structures were found in all three systems, with a maximum $T_{\mathrm{c}}$ of $235 \mathrm{~K}$ in $\mathrm{CaH}_{6}$ and $21 \mathrm{~K}$ in $\mathrm{BH}$, both above $150 \mathrm{GPa}$, and $6 \mathrm{~K}$ in $\mathrm{CaB}$ at $30 \mathrm{GPa}$ [39,64,65].

In this study, to obtain the phase diagram as a function of pressure, we sampled the full ternary $\mathrm{Ca}-\mathrm{B}-\mathrm{H}$ phase space with variable-composition structural searches at fixed pressures $(0,50,100$, and $300 \mathrm{GPa})$ to identify the compositions stable at room, intermediate, and extreme pressures. More than 4000 unique structures were sampled for each pressure. The lowest-enthalpy structures for each composition identified in these runs were then relaxed at intermediate pressures to obtain the phase diagram. Further details can be found in the Appendix.

Figure 1 shows the ternary convex hulls for the four pressures studied. The stable and metastable compositions are shown as circles and squares, respectively, while metal/nonmetal character is indicated by full/empty symbols. The figures highlight a gradual transition from low to high-pressure stoichiometries, and from insulating to metal behavior.

At ambient pressure, our predictions agree with previous experiments and calculations [39,57,63-67]. At room 

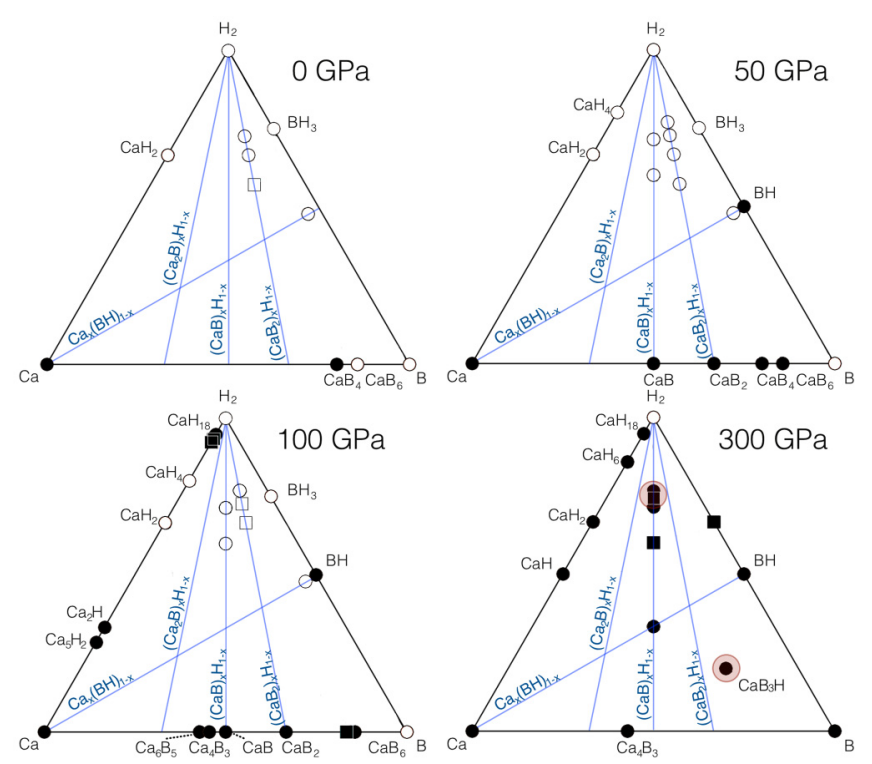

FIG. 1. Convex hull diagrams for calcium, boron and hydrogen at $0,50,100$, and $300 \mathrm{GPa}$ predicted from evolutionary crystal structure prediction calculations. Thermodynamically stable and metastable structures are shown as circles and squares, while empty and filled symbols represent insulating and metallic structures, respectively. Compositions within $50 \mathrm{meV} /$ atom from the hull are considered metastable. Blue lines indicate lines where additional structural searches were carried out. The compositions circled in red, $\mathrm{CaBH}_{6}, \mathrm{Ca}_{2} \mathrm{~B}_{2} \mathrm{H}_{13}$, and $\mathrm{CaB}_{3} \mathrm{H}$, exhibit a finite $T_{\mathrm{c}}$.

pressure, the stable (or weakly metastable) phases lie along the $\mathrm{Ca}\left(\mathrm{BH}_{x}\right)_{2}$ line. Their structures comprise $\mathrm{BH}_{x}^{-1}$ anions, with hydrocarbon-like motifs, and $\mathrm{Ca}$ cations in the interstitials: $\mathrm{Ca}\left(\mathrm{BH}_{2}\right)_{2}$ (polyethylene), $\mathrm{Ca}\left(\mathrm{BH}_{3}\right)_{2}$ (ethane), and
$\mathrm{Ca}\left(\mathrm{BH}_{4}\right)_{2}$ (methane). In addition, there is a stable $\mathrm{CaB}_{12} \mathrm{H}_{12}$ phase, containing $\mathrm{B}_{12} \mathrm{H}_{12}^{2-}$ icosahedra $[63,68]$. The crystal structure of $\mathrm{Ca}\left(\mathrm{BH}_{4}\right)_{2}$ is shown in the top left panel of Fig. 2.

At $50 \mathrm{GPa}$, most compositions stable at ambient pressure remain on the convex hull, with structures which maintain the motifs observed at ambient pressures. In addition, new compositions appear along the $\mathrm{Ca}\left(\mathrm{BH}_{x}\right)_{2}$ and $\mathrm{Ca}\left(\mathrm{BH}_{x}\right)$ lines, as hydrogen is trapped in the open molecular structures stable at room pressure. In $\mathrm{Ca}\left(\mathrm{BH}_{5}\right)_{2}$, interstitial $\mathrm{H}_{2}$ molecules are trapped between $\mathrm{BH}_{4}$ tetrahedra, in $P 2_{1} / m \mathrm{CaBH}_{3}$ a single $\mathrm{H}$ atom occupies the interstitial sites among $\mathrm{Ca}$ tetrahedra, which are sandwiched between $\mathrm{BH}_{2}$ linear chains, while in $F \overline{4} 3 m \mathrm{CaBH}_{5}$ a single $\mathrm{H}$ atom is trapped in the interstitial sites between $\mathrm{BH}_{4}$ anionic tetrahedra-bottom left panel of Fig. 2. Trapping of atomic or molecular hydrogen is observed at high pressures also in many covalent hydrides [4].

At $100 \mathrm{GPa}$, phases on the $\mathrm{Ca}\left(\mathrm{BH}_{x}\right)_{2}$ line become unstable, while $\mathrm{CaBH}_{3}$ and $\mathrm{CaBH}_{5}$, on the $(\mathrm{CaB}) \mathrm{H}_{x}$ line, remain stable. In addition, $\mathrm{CaB}_{12} \mathrm{H}_{12}$ has a structural phase transition towards a phase with $R \overline{3} m$ space group, in which the $\mathrm{B}_{12} \mathrm{H}_{12}^{2-}$ icosahedra are more densely packed. All structures are still insulating at this pressure, but metallization sets in by band overlap immediately after in $\mathrm{CaBH}_{5}$

None of the $\mathrm{Ca}\left(\mathrm{BH}_{x}\right)_{2}$ ambient-pressure compositions survives up to $300 \mathrm{GPa}$, where the pressure is so high that it leads to a complete rearrangement of the bonds: boron and hydrogen now form interconnected, dense networks, and the structures are all metallic. The stable high-pressure crystal structures are shown in the right panel of Fig. 2.

$\mathrm{CaB}_{3} \mathrm{H}$ is characterized by buckled triangular boron layers, similar to those predicted in Ref. [65] for pure boron, alternated with $\mathrm{CaH}$ planes, so that the $\mathrm{Ca}$ atoms fits into the valleys of the $\mathrm{B}$ layers. $\mathrm{CaBH}$ is constituted by infinite $\mathrm{B}-\mathrm{H}$ buckled chains in the $z$ direction, parallel to $\mathrm{Ca}$ chains.
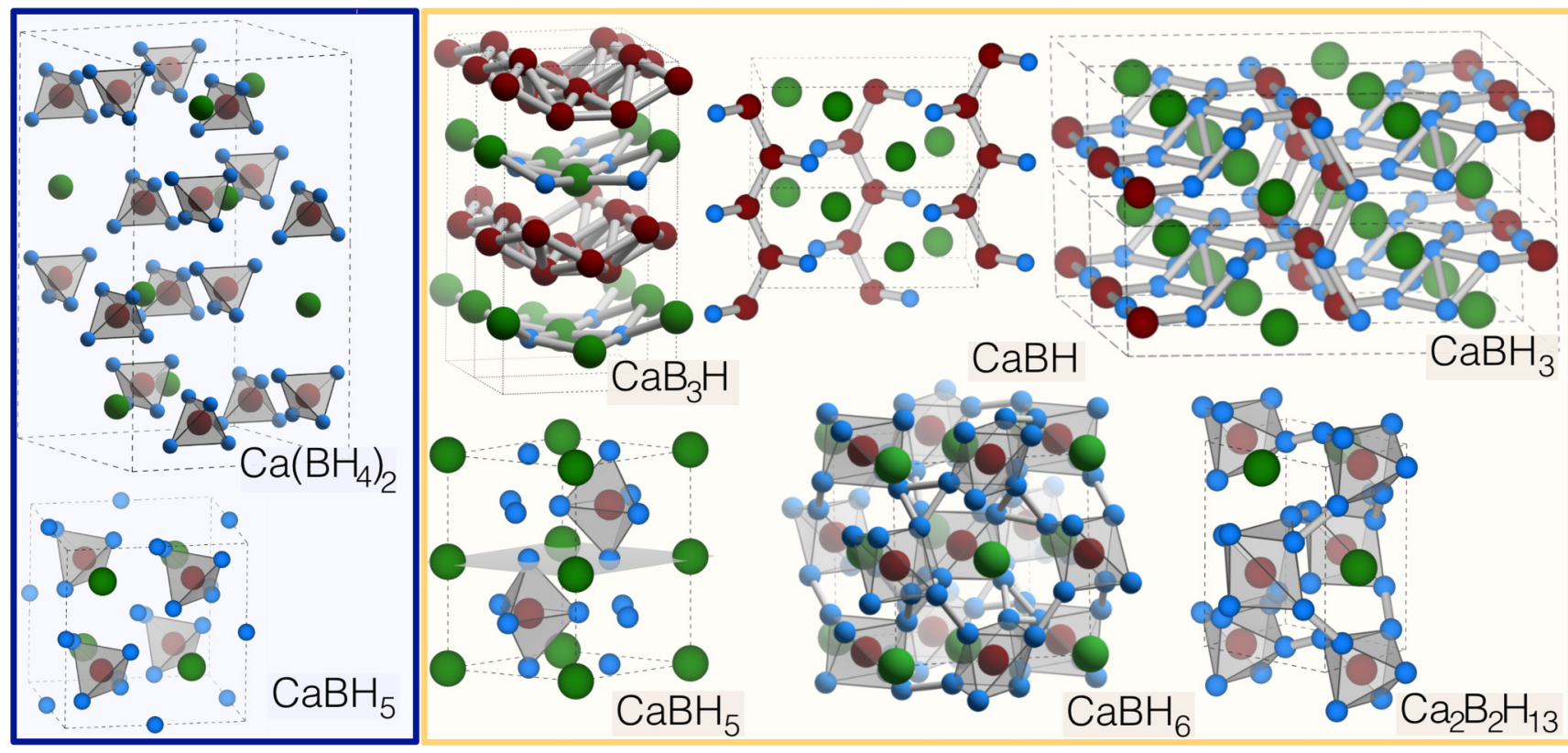

FIG. 2. (Left) Crystal structures of the $F d d d$ phase of $\mathrm{Ca}\left(\mathrm{BH}_{4}\right)_{2}$ at $0 \mathrm{GPa}$, and $F \overline{4} 3 m$ of $\mathrm{CaBH}_{5}$ at $50 \mathrm{GPa}$. (Right) Crystal structures of high-pressure $A m m 2$ phase of $\mathrm{CaB}_{3} \mathrm{H}$, Ima 2 phase of $\mathrm{CaBH}, C 2 / m$ phase of $\mathrm{CaBH}_{3}, P 63 m m c$ phase of $\mathrm{CaBH}_{5}$, metastable $P a \overline{3}$ phase of $\mathrm{CaBH}_{6}$, and $\mathrm{Pm}$ phase of $\mathrm{Ca}_{2} \mathrm{~B}_{2} \mathrm{H}_{13}$. Calcium, boron, and hydrogen are shown as green, red, and turquoise spheres, respectively. 


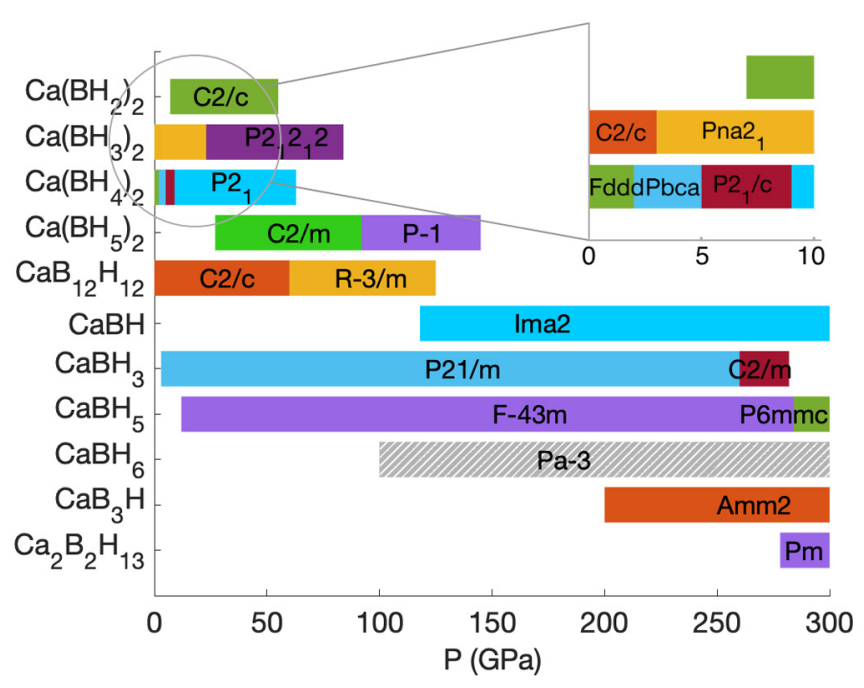

FIG. 3. Phase diagram for intermediate compositions as a function of pressure. The space group for each phase is indicated on top of the bars. The slashed pattern indicates the dynamical stability of a high-symmetry $\mathrm{CaBH}_{6}$ structure, which is thermodynamically unstable. The inset shows phase transitions between low-pressure structures for $\mathrm{Ca}\left(\mathrm{BH}_{x}\right)_{2}$ compositions.

$\mathrm{Ca}_{2} \mathrm{~B}_{2} \mathrm{H}_{13}$ exhibits a structure with distorted $\mathrm{B}-\mathrm{H}$ octahedra and triangular bipyramids, and interstitial $\mathrm{H}_{2}$ molecules. $\mathrm{CaBH}_{5}$ undergoes a structural phase transition from the $F \overline{4} m$ phase identified at $50 \mathrm{GPa}$ and shown in the left panel of Fig. 2, to a phase with space group $P 6_{3} m m c$, in which the interstitial $\mathrm{H}$ atoms are incorporated in the $\mathrm{B}-\mathrm{H}$ sublattice to form $\mathrm{BH}_{5}$ triangular bipyramids.

In addition to these thermodynamically stable phases, among H-rich compositions we also identified a highsymmetry structure with space group $P a \overline{3}$ for $\mathrm{CaBH}_{6}$, lying about $100 \mathrm{meV} /$ atom above the hull. This structure comprises $\mathrm{BH}_{6} 6$-vertex antiprisms, with hydrogen at the vertexes and boron in the middle. The nearest-neighbor $\mathrm{H}-\mathrm{H}$ distance (1.2 $\AA$ ) is close to that of atomic hydrogen [69], suggesting the formation of a metallic $\mathrm{H}$ sublattice.

Given the empirical correlation between $\mathrm{H}-\mathrm{H}$ interatomic distances and high- $T_{\mathrm{c}}$ superconductivity in high-pressure hydrides, we decided to include this structure in our pool of potential high- $T_{\mathrm{c}}$ superconductors.

The phase diagram in Fig. 3 summarizes the stability range of the different $\mathrm{Ca}-\mathrm{B}-\mathrm{H}$ structures as a function of pressure. As it can be appreciated, several phases which appear on the $300 \mathrm{GPa}$ convex hull remain stable up to much lower pressures.

There is a rather clear separation of the phase diagram between a low- and a high-pressure regime, setting in at around $100 \mathrm{GPa}$. The group of low-pressure phases comprises $\mathrm{Ca}\left(\mathrm{BH}_{x}\right)_{2}(x=2,3,4)$ compositions, as well as $\mathrm{CaB}_{12} \mathrm{H}_{12}$; as shown previously, all these phases form molecular, insulating crystals. A series of structural transitions occurs between 0 and $50 \mathrm{GPa}$, due to the progressive rearrangement of $\mathrm{BH}_{x}$ units in the crystal. At pressures between 10 and $20 \mathrm{GPa}$, hydrogen starts to get trapped in molecular or atomic form in these open structures, stabilizing $\mathrm{H}$-rich stoichiometries, such as $\mathrm{Ca}\left(\mathrm{BH}_{5}\right)_{2}, \mathrm{Ca}\left(\mathrm{BH}_{3}\right)$ and $\mathrm{Ca}\left(\mathrm{BH}_{5}\right)$.
Additional structures along the $\mathrm{Ca}(\mathrm{BH})_{x}$ line become stable above $100 \mathrm{GPa}$ : in these phases, the low-P motifs of the B-H sublattice, analogous to hydrocarbons, are replaced by new ones, in which boron is coordinated to five or more hydrogen atoms. This qualitative transition of the B-H sublattice behavior is accompanied by the onset of metallic behavior. In fact, all structures we report at $300 \mathrm{GPa}$ are metallic in their whole stability range.

\section{ELECTRONIC STRUCTURE}

We now discuss the electronic properties of the structures in the Ca-B-H high-pressure phase diagram. Structures which are stable up to $100 \mathrm{GPa}$ are of no interest for superconductivity, since they are insulating. Hence, here we discuss the main features of their electronic structure only briefly, and collect the plots of the relative Densities of States (DOS) in the Supplemental Material [58].

Room pressure structures, characterized by a disconnected lattice of hydrocarbon-like, $\mathrm{BH}_{x}^{-}$anions, exhibit molecularlike DOS's, with sharp peaks of mixed $\mathrm{B}$ and $\mathrm{H}$ character, and band gaps ranging from $2.6 \mathrm{eV}$ for $\mathrm{Ca}\left(\mathrm{BH}_{2}\right)_{2}$ to $5 \mathrm{eV}$ for $\mathrm{Ca}\left(\mathrm{BH}_{4}\right)_{2}$. $\mathrm{Ca} 4 s$ and $3 d$ states lie at the bottom the conduction band; $\mathrm{Ca}$ is fully ionized, as confirmed by a Bader charge analysis, which assigns a total charge of about +1.5 $e$ to it. As pressure is increased to $50 \mathrm{GPa}$, the peaks of the DOS broaden and merge into a continuous band; the bonding/antibonding gaps decrease to $1.8 \mathrm{eV}$ for $\mathrm{Ca}\left(\mathrm{BH}_{2}\right)_{2}$, and $3.4 \mathrm{eV}$ for $\mathrm{Ca}\left(\mathrm{BH}_{4}\right)_{2}$. The new $\mathrm{Ca}\left(\mathrm{BH}_{5}\right)_{2}, \mathrm{CaBH}_{3}$ and $\mathrm{CaBH}_{5}$ phases are all insulating, with gaps of $4.1,1.6$, and $2.3 \mathrm{eV}$, respectively.

At $100 \mathrm{GPa}$ the reported phases for $\mathrm{Ca}\left(\mathrm{BH}_{3}\right)_{2}, \mathrm{Ca}\left(\mathrm{BH}_{4}\right)_{2}$, $\mathrm{Ca}\left(\mathrm{BH}_{5}\right)_{2}$ and $\mathrm{CaB}_{12} \mathrm{H}_{12}$ are still insulating, with calculated gaps of $1.6,0.5,1.8$, and $2.6 \mathrm{eV}$, respectively, $\mathrm{CaBH}_{3}$ exhibits a gap of $0.4 \mathrm{eV}, \mathrm{CaBH}_{5}$ is semimetallic.

As shown in Fig. 3, phases resulting from a the full rearrangement of the $\mathrm{B}-\mathrm{H}$ sublattice at high pressures gradually appear on the convex hull only above $100 \mathrm{GPa}$. We discuss their electronic properties at a common pressure of $300 \mathrm{GPa}$, where high- $P$ structures are stable for all compositions. In Fig. 4, we show the electronic DOS projected onto the atomic orbitals for $\mathrm{CaB}_{3} \mathrm{H}, \mathrm{CaBH}, \mathrm{CaBH}_{3}, \mathrm{CaBH}_{5}, \mathrm{CaBH}_{6}$, and $\mathrm{Ca}_{2} \mathrm{~B}_{2} \mathrm{H}_{13}$. The energies have been rescaled with respect to the Fermi energy. The behavior of the DOS in the valence region depends strongly on the relative boron and hydrogen content: the $\mathrm{CaB}_{3} \mathrm{H}$ and $\mathrm{CaBH}$ DOS are dominated by electronic states with $\mathrm{B}$ character. On the other hand, the hydrogen-rich phases exhibit a strong $\mathrm{B}$ and $\mathrm{H}$ hybridization over the whole -20 to $5 \mathrm{eV}$ range; furthermore, $\mathrm{Ca}-4 s$ states are spread over the valence and conduction band, so that $\mathrm{Ca}$ is only partly ionized, with an average net Bader charge of +0.9 . In $\mathrm{CaBH}_{5}$ the valence band exhibits $\mathrm{B}$ and $\mathrm{H}$ character with the exception of energies near the Fermi level, where around half of the contribution to the total DOS comes from states with $\mathrm{Ca}-3 d$ character.

In $\mathrm{CaBH}_{6}$ and $\mathrm{Ca}_{2} \mathrm{~B}_{2} \mathrm{H}_{13}$, the occupied states mostly have $\mathrm{B}$ and $\mathrm{H}$ character; the $\mathrm{B}-$ and $\mathrm{H}$ - projected DOS follow each other rather closely, which indicates that the two are covalently bonded, while the $\mathrm{Ca}-3 d$ states remain empty, and lie around $2 \mathrm{eV}$ above the Fermi energy. 


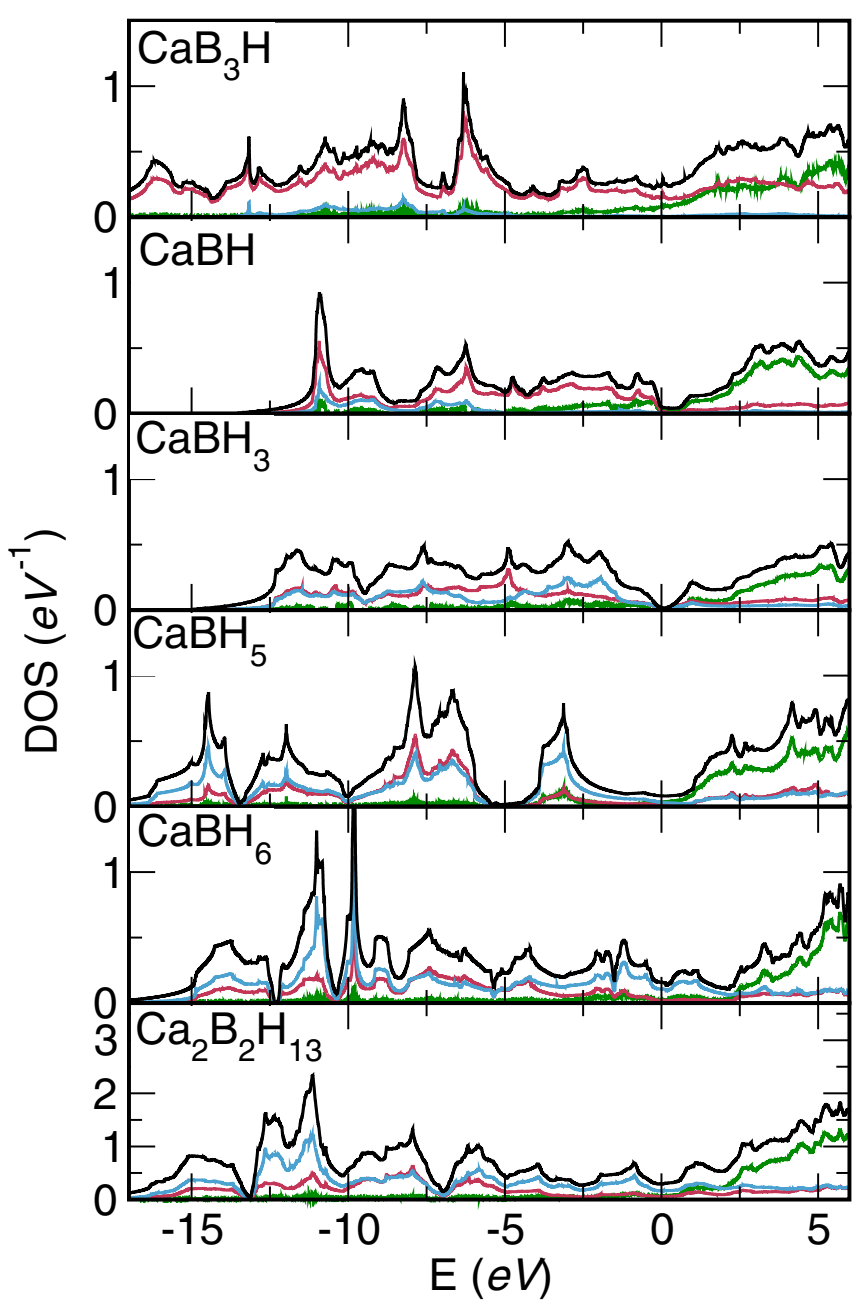

FIG. 4. Calculated total and projected DOS for stable and metastable calcium boron hydrides at $300 \mathrm{GPa}$. Black, red, turquoise, and green lines represent the total DOS, and its projection onto $\mathrm{B}, \mathrm{H}$, and $\mathrm{Ca}$, respectively. The energy range is shifted with respect to the Fermi level. The DOS is in units of states per eV per formula unit. From top to bottom: $\mathrm{Amm} 2-\mathrm{CaB}_{3} \mathrm{H}, \mathrm{Ima} 2-\mathrm{CaBH}, \mathrm{C} 2 / m-\mathrm{CaBH}_{3}$, $P 6_{3} m m c-\mathrm{CaBH}_{5}, P a \overline{3}-\mathrm{CaBH}_{6}$, and the $P m-\mathrm{Ca}_{2} \mathrm{~B}_{2} \mathrm{H}_{13}$.

Note that the Perdew-Burke-Ernzerhof (PBE) functional employed in our calculations is known to underestimate the band gap, and hence the transition pressure. To obtain a more reliable estimate we computed the band gap as a function of pressure for the $P 63 m m c$ phase of $\mathrm{CaBH}_{5}$ using a HeydScuseria-Ernzerhof (HSE) hybrid functional [70]. The band gap thus estimated is about $1 \mathrm{eV}$ larger than the PBE at all pressures. This increases the onset of metallization by around $60 \mathrm{GPa}$.

\section{SUPERCONDUCTIVITY}

With the exception of $\mathrm{CaBH}_{3}$, which is a semimetal with a very small DOS at the Fermi level, all high-pressure Ca$\mathrm{B}-\mathrm{H}$ phases are metallic, and hence have the potential to be high- $T_{\mathrm{c}}$ superconductors. In order to quantitatively assess their superconducting properties, we carried out calculations of their electron-phonon properties, using density functional

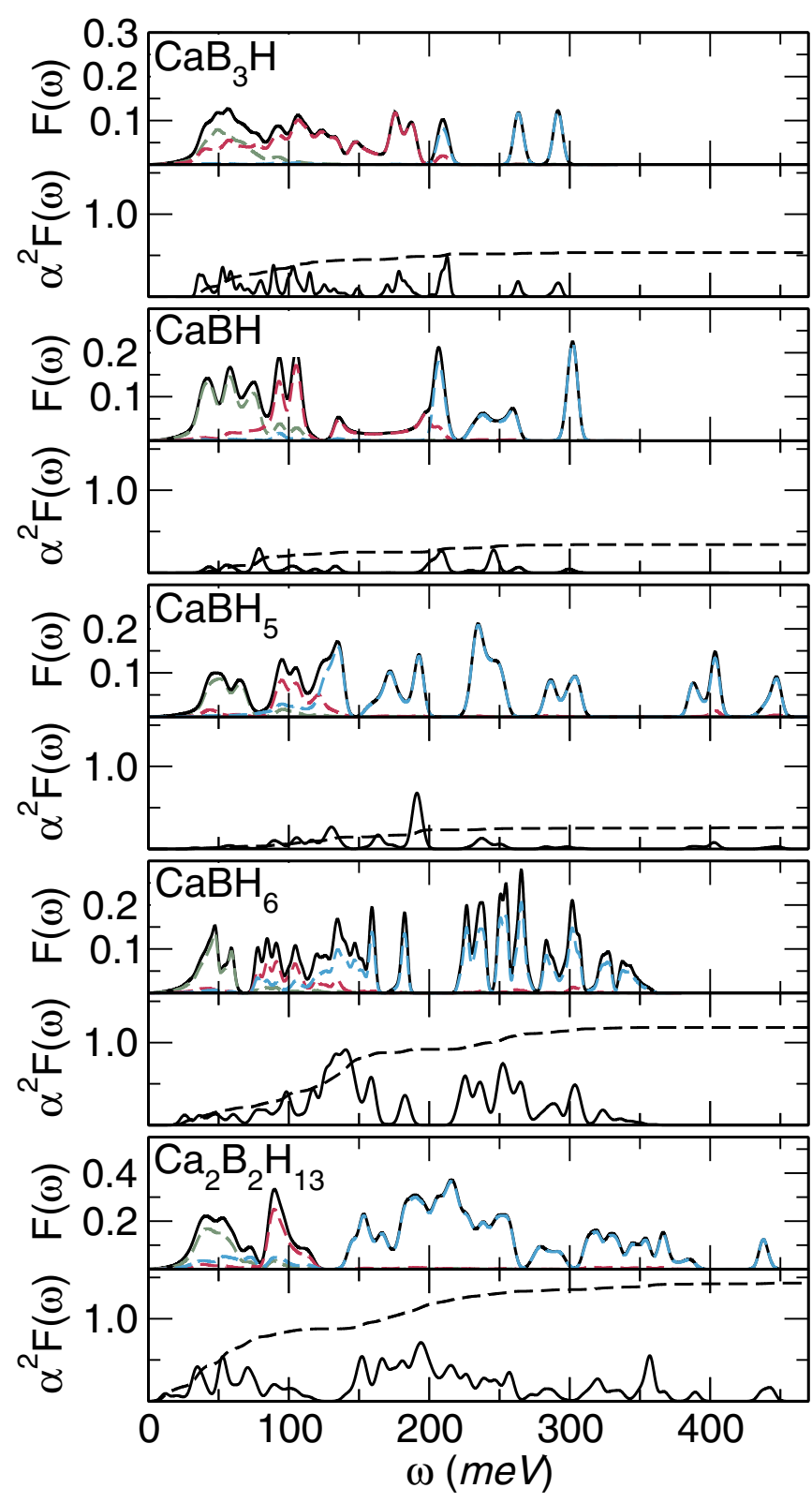

FIG. 5. Atom-projected phonon DOS $[F(\omega)]$, Eliashberg function $\left[\alpha^{2} F(\omega)\right.$, solid lines], and frequency-dependent $e$-ph coupling constant $[\lambda(\omega)$, dashed lines] calculated at $300 \mathrm{GPa}$ for the same six high-pressure phases as in Fig. 4. The total phonon DOS is shown in black, and its projections on $\mathrm{Ca}, \mathrm{B}$, and $\mathrm{H}$, are shown in green, red, and blue, respectively.

perturbation theory, as implemented in the plane-wave pseudopotential code QUANTUM ESPRESSO [12,54,71].

The top panels of Fig. 5 show the calculated total and partial phonon densities of states of $\mathrm{CaB}_{3} \mathrm{H}, \mathrm{CaBH}, \mathrm{CaBH}_{5}$, $\mathrm{CaBH}_{6}$, and $\mathrm{Ca}_{2} \mathrm{~B}_{2} \mathrm{H}_{13}$, with the same color coding as for the electronic DOS's in Fig. 4.

For all structures there is a net separation of phonon spectra in three regions: a low-energy one, extending from 0 to $75 \mathrm{meV}$, dominated by modes of $\mathrm{Ca}$ character, an intermediate one, from 75 to $175 \mathrm{meV}$, with modes of mixed B and $\mathrm{H}$ character, and a high-energy region, from 175 up to $300 \mathrm{meV}$, characterized by B-H stretching modes. Moreover, 
TABLE I. Normal and superconducting state properties of the six high-pressure Ca-B-H phases in Figs. 4 and 5. Space group, number of formula units, unit cell volume; DOS at the Fermi level $N\left(E_{F}\right)$, Hydrogen fraction of the total DOS at the Fermi level $\left[N_{H} / N\left(E_{F}\right)\right]$, electronphonon coupling constant $(\lambda)$, effective $e$-ph matrix element $\left[\lambda / N\left(E_{F}\right)\right]$, logarithmic-average phonon frequency $\left(\omega_{\log }\right)$, McMillan-Allen-Dynes superconducting $T_{\mathrm{c}}$ [Eq. (1)], with $\mu^{*}=0.10$ and 0.15 . The DOS at the Fermi level is expressed in states/spin and is rescaled by volume to allow for an easier comparison between different pressures.

\begin{tabular}{|c|c|c|c|c|c|c|c|c|c|c|c|}
\hline & $\begin{array}{c}\mathrm{P} \\
(\mathrm{GPa})\end{array}$ & Space group & f.u. & $\begin{array}{l}\text { Volume } \\
\left(\AA^{3}\right)\end{array}$ & $\left(10^{2}\right.$ & $\begin{array}{l}N\left(E_{F}\right) \\
\left.\text { st eV } \mathrm{eV}^{-1} \AA^{-3}\right)\end{array}$ & $N_{H} / N\left(E_{F}\right)$ & $\lambda$ & $\begin{array}{c}\lambda / N\left(E_{F}\right) \\
\left(10^{-2} \mathrm{eV} \AA^{3}\right)\end{array}$ & $\begin{array}{c}\omega_{\log } \\
(\mathrm{meV})\end{array}$ & $\begin{array}{l}T_{\mathrm{c}} \\
(\mathrm{K})\end{array}$ \\
\hline $\mathrm{CaB}_{3} \mathrm{H}$ & 300 & Amm 2 & 2 & 43.6 & & 1.08 & 0.02 & 0.48 & 0.44 & 78 & $4-7$ \\
\hline $\mathrm{CaBH}_{5}$ & 300 & P6mmc & 2 & 39.9 & & 0.41 & 0.40 & 0.26 & 0.63 & 131 & $\leqslant 0.1$ \\
\hline $\mathrm{CaBH}_{6}$ & 100 & $P a \overline{3}$ & 4 & 119.7 & & 0.57 & 0.73 & 1.93 & 3.33 & 70 & $100-114$ \\
\hline $\mathrm{CaBH}_{6}$ & 200 & $\mathrm{~Pa} \overline{3}$ & 4 & 97.6 & & 0.64 & 0.67 & 1.27 & 1.99 & 106 & $97-117$ \\
\hline $\mathrm{Ca}_{2} \mathrm{~B}_{2} \mathrm{H}_{13}$ & 200 & $P m$ & 1 & 50.2 & & 0.59 & 0.51 & 1.23 & 2.10 & 59 & $51-62$ \\
\hline $\mathrm{Ca}_{2} \mathrm{~B}_{2} \mathrm{H}_{13}$ & 300 & $\mathrm{Pm}$ & 1 & 43.9 & & 0.67 & 0.55 & 1.37 & 2.07 & 74 & $75-89$ \\
\hline
\end{tabular}

hydrogen-rich structures exhibit a further high-energy band, above $300 \mathrm{meV}$, which involves purely $\mathrm{H}$ vibrations and is not present in $\mathrm{CaB}_{3} \mathrm{H}$ and $\mathrm{CaBH}$.

The bottom panels of the figure show, for each Ca-B-H phase, the calculated Eliashberg (electron-phonon) spectral function, which is essentially a phonon density of states, weighted by the electron-phonon matrix elements for states at the Fermi level [72]. Comparing its shape with the phonon DOS one can obtain a direct information on the distribution of the $e$-ph coupling over the phonon spectrum. In all cases, the Eliashberg function follows quite closely the phonon DOS, indicating that the $e$-ph coupling is distributed almost evenly on all vibrational modes, in analogy with other high- $T_{\mathrm{c}}$ highpressure hydrides $[4,38,41,73]$.

From the Eliashberg function, we obtained the logarithmic average phonon frequency $\omega_{\log }$ and the $e$-ph coupling constant $\lambda$, which appear in the McMillan-Allen-Dynes formula for the critical temperature $[13,74]$ :

$$
T_{c}=\frac{\omega_{\log }}{1.2} \exp \left(\frac{-1.04(1+\lambda)}{\lambda\left(1-0.62 \mu^{*}\right)-\mu^{*}}\right)
$$

and measure respectively the average effective frequency of the phonons participating in the superconducting pairing and the intensity with which they couple to electrons at the Fermi level.

These quantities are reported in Table I for all five Ca-B$\mathrm{H}$ compounds shown in Fig. 5. The last column of the table shows also the $T_{\mathrm{c}} \mathrm{s}$, obtained from Eq. (1) for a standard choice of the Morel-Anderson pseudopotential, $\mu^{*}=0.1$.

For only two out of five structures we predict a $T_{\mathrm{c}}$ exceeding liquid nitrogen boiling point: $\mathrm{CaBH}_{6}\left(T_{\mathrm{c}}=119 \mathrm{~K}\right)$ and $\mathrm{Ca}_{2} \mathrm{~B}_{2} \mathrm{H}_{13}\left(T_{\mathrm{c}}=89 \mathrm{~K}\right)$. Both are hydrogen-rich, highsymmetry phases. In both cases, superconductivity survives up to lower pressures, with sizable $T_{\mathrm{c}} \mathrm{s}$.

Boron-rich $\mathrm{CaB}_{3} \mathrm{H}$ also exhibits a finite, although much lower $T_{\mathrm{c}}(7 \mathrm{~K})$. This is not surprising since an inspection of the electronic DOS in Fig. 4 shows that the contribution of $\mathrm{H}$ states is negligible in the whole valence region, and the fraction of $\mathrm{H}$ states at the Fermi level, $N_{H} / N_{\text {tot }}$, is as low as $2 \%$. Essentially, the superconducting pairing is dominated by electronic and vibrational states of the boron sublattice, determining an intermediate $e$-ph coupling constant $(\lambda=$
0.48), and an $\omega_{\log }$ which is sensibly larger than in superconducting borides, but about half than high- $T_{\mathrm{c}}$ hydrides [16]

The other two metallic calcium borohydrides stable at high-pressure, $\mathrm{CaBH}$ and $\mathrm{CaBH}_{5}$, should not be superconducting according to our calculations. Both exhibit a DOS at the Fermi level which is comparable to that of other highpressure hydrides; note that the value of the DOS in Table I has been rescaled with the unit cell volume, in order to allow for a meaningful comparison across structures with different compositions and stability pressures.

However, the value of the parameter $\lambda / N\left(E_{F}\right)$, is remarkably smaller than in the two high- $T_{\mathrm{c}} \mathrm{Ca}-\mathrm{B}-\mathrm{H}$ phases. This parameter essentially measures the intrinsic coupling between electrons at the Fermi level and lattice vibrations. In $\mathrm{CaBH}$, its low value can be explained in terms of a very small fraction of $\mathrm{H}$-derived electronic states at the Fermi level $\left(N_{H} / N_{\text {tot }}=0.06\right)$, leading to ineffective $e$ - $p h$ coupling. The same argument cannot be applied in $\mathrm{CaBH}_{5}$, where about half of the electronic states at the Fermi level have $\mathrm{H}$ orbital character.

To understand why in $\mathrm{CaBH}_{5}$ electrons at the Fermi level couple so little to phonons, despite a strong $\mathrm{H}$ orbital character, it is useful to inspect the so-called local density of states (LDOS):

$$
N(E, \mathbf{r})=\sum_{n} \int \frac{d^{3} k}{(2 \pi)^{3}} \delta\left(E-\varepsilon_{n \mathbf{k}}\right)\left|\psi_{n \mathbf{k}}(\mathbf{r})\right|^{2},
$$

where $\psi_{n}(\mathbf{k})(\mathbf{r})$ and $\varepsilon_{n \mathbf{k}}$ are the Kohn-Sham eigenfunctions and eigenvalues of the system. The LDOS permits to visualize the real-space distribution of the electronic states at a given energy $E$. If computed for $E=E_{F}$, it permits to visualize the real-space distribution of electrons, which participate in the superconducting pairing [75].

Figure 6 shows isosurfaces and isocontours of the LDOS at $E_{F}$ for high- $T_{\mathrm{c}} \mathrm{CaBH}_{6}$ (top) and nonsuperconducting $\mathrm{CaBH}_{5}$ (bottom). In $\mathrm{CaBH}_{6}$, the electronic charge is concentrated on the B-H sublattice, and hence is highly susceptible to B-H bond-stretching vibrations that dominate the intermediate and high-frequency part of the phonon spectrum, giving rise to large $e$-ph matrix elements. In $\mathrm{CaBH}_{5}$, on the other hand, a considerable fraction of the charge is concentrated around $\mathrm{Ca}$ 


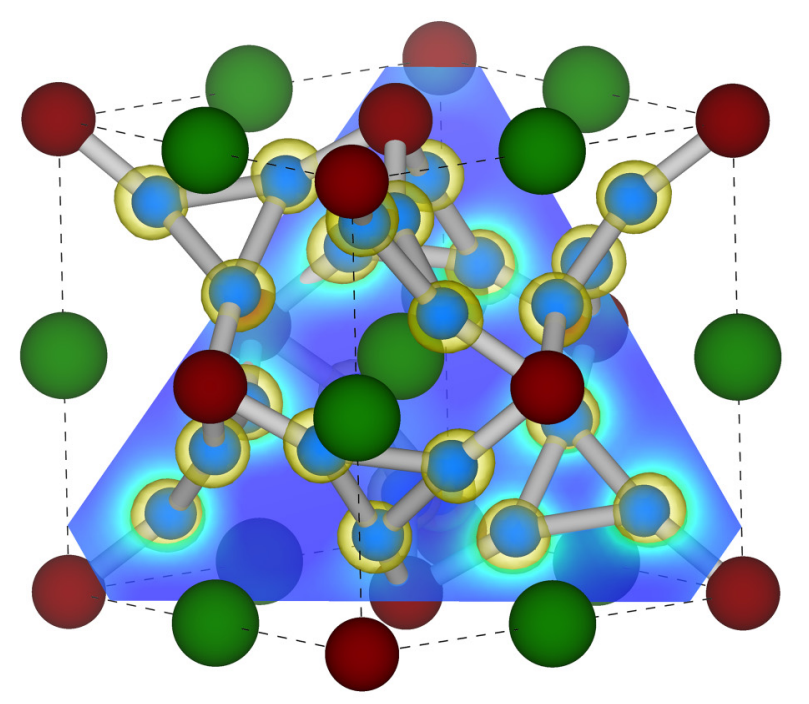

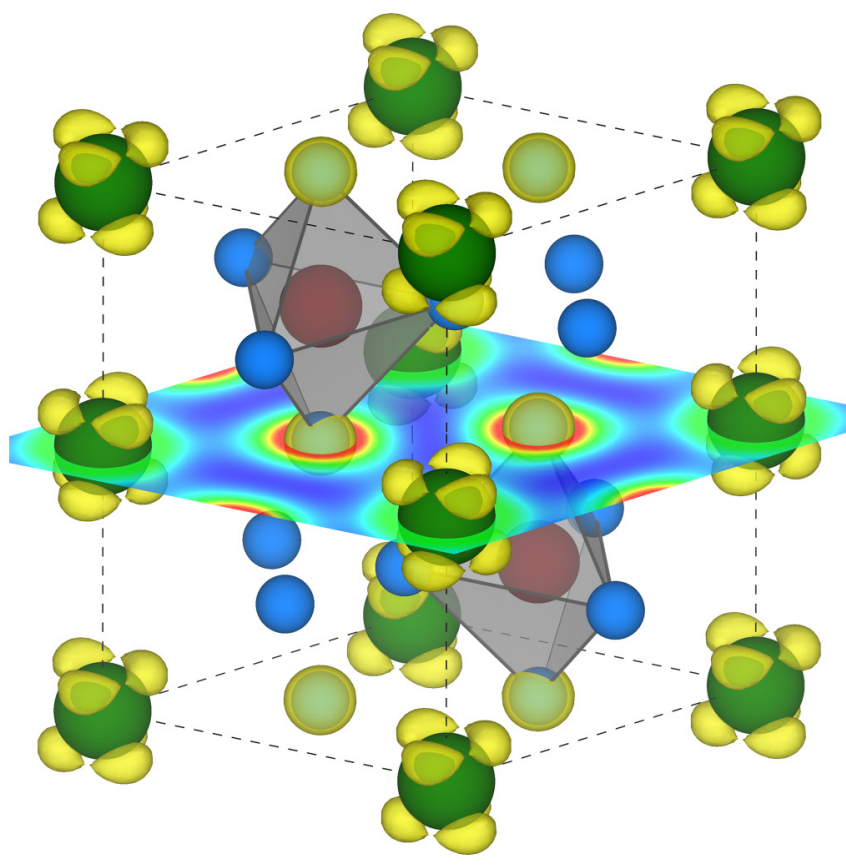

FIG. 6. LDOS for $\mathrm{CaBH}_{6}$ (top) and $\mathrm{CaBH}_{5}$ (bottom). The yellow isosurface represents the LDOS distribution at $50 \%$ of its maximum value. The lattice plane shows the LDOS along the 001 plane of $\mathrm{CaBH}_{5}$ and $1 \overline{1} 1$ plane of $\mathrm{CaBH}_{6}$. The color scale on the lattice plane goes from the minimum (blue) to the maximum (red) value of the LDOS. The $\mathrm{BH}_{6}$ antiprisms of $\mathrm{CaBH}_{6}$ are not shown for visual clarity.

atoms, with a distribution typical of $d$ planar orbitals. The spatial overlap of these electronic states with neighboring $\mathrm{B}$ or $\mathrm{H}$ atoms is quite small, and hence their coupling to $\mathrm{B}-\mathrm{H}$ vibrations is strongly suppressed with respect to $\mathrm{CaBH}_{6}$. The same picture is confirmed by "fat-band" plots, reported in the Supplemental Material [58], which show that Ca-3d-derived states dominate one of the two pockets of the Fermi surface of $\mathrm{CaBH}_{5}$, but give none or negligible contributions to the electronic states that form the Fermi surfaces of $\mathrm{CaBH}_{6}$ and $\mathrm{Ca}_{2} \mathrm{~B}_{2} \mathrm{H}_{13}$. Hence, $\mathrm{Ca} d$ orbitals play an essential role in determining the superconducting properties of different calcium borohydrides.

\section{CONCLUSIONS}

In this work, we have studied $a b$ initio the high-pressure superconducting phase diagram of the ternary calcium-boronhydrogen system, using evolutionary crystal structure prediction and density functional perturbation theory, with the aim of identifying new high- $T_{\mathrm{c}}$ high-pressure hydrides. The original hypothesis of this work was that the combination of hydrogen with a weak $(\mathrm{Ca})$ and a strong $(\mathrm{B})$ hydride former at high pressures could provide a large playground of covalent metallic crystals, to improve the superconducting properties, compared to binary hydrides.

Our structural searches have shown that the Ca-B-H phase diagram can be clearly split in two regions: the lowpressure region, extending from room pressure to approximately $100 \mathrm{GPa}$, is characterized by closed-shell, insulating phases, forming open molecular structures. As pressure is increased, hydrogen is gradually incorporated into the B$\mathrm{H}$ lattice, stabilizing hydrogen-rich compositions. At around $100 \mathrm{GPa}$, a different high-pressure regime sets in, following the rearrangement of boron and hydrogen bonds into dense sublattices; the new structures that form gradually become metallic at higher pressures. Among these high-pressure structures, we identify two phases, with unusually high $\mathrm{H}: \mathrm{B}$ ratios: $\mathrm{CaBH}_{6}$ and $\mathrm{Ca}_{2} \mathrm{~B}_{2} \mathrm{H}_{13}$, characterized by five- and sixcoordinated boron atoms, surrounded by hydrogen. For these two phases at $300 \mathrm{GPa}$, we estimate a $T_{\mathrm{c}}$ of 119 and $89 \mathrm{~K}$, respectively, which survive to lower pressures. These $T_{\mathrm{c}} \mathrm{s}$ are higher than those reported for the stable phases of the binary B-H system [65], and comparable to those predicted in the related Li-B-H system. [51] We also show that a third hydrogen-rich phase, $\mathrm{CaBH}_{5}$, despite being metallic, is nonsuperconducting; this can be attributed to a dominant contribution of $\mathrm{Ca} d$ states to the electronic states at the Fermi surface, which couple poorly to $\mathrm{B}$ and $\mathrm{H}$ phonons. In this work, we neglected anharmonic effects which are known to affect superconducting properties of hydrides [35,73] since this level of detail was beyond the scope of our exploratory work.

On one hand, our results confirm that ternary hydrides are a promising venue to search for high-temperature conventional superconductivity; on the other hand, they also highlight that details of electronic structure and chemistry may play a key role in determining the final superconducting $T_{\mathrm{c}}$. In fact, we find that high-pressure ternary $\mathrm{Ca}-\mathrm{B}-\mathrm{H}$ covalent metallic structures are not competitive with the best covalent hydrides, such as $\mathrm{SH}_{3}$, for two reasons: (1) since boron is very electronegative, closed-shell, insulating structures survive up to high pressures, and metalization slowly sets in only after $100 \mathrm{GPa}$. (2) Since the $d$ orbitals of Ca lie close to the valence, at high pressure they may influence the behavior of valence electronic states in unpredictable ways, acting very differently from other alkali metals and alkaline earths.

Our study hence suggests that searches for high- $T_{\mathrm{c}}$ superconductivity in ternary hydrides should be oriented toward compounds containing elements less electronegative than boron, such as aluminum and silicon, where bonds can be more easily broken and reformed. Recent experimental work, which has yet to be confirmed, seems to suggest that superconductivity well above room temperature may occur 
in ternary or quaternary hydrides, which is an interesting research direction for computational materials science [76].

\section{ACKNOWLEDGMENTS}

The authors acknowledge computational resources from the dCluster of the Graz University of Technology and the VSC3 of the Vienna University of Technology, and support through the FWF, Austrian Science Fund, Project P 30269N36 (Superhydra). L. B. acknowledges funding through Progetto Ateneo Sapienza 2017-18-19 and computational Resources from CINECA, proj. Hi-TSEPH.

\section{APPENDIX: COMPUTATIONAL DETAILS}

\section{Structural prediction}

To construct the $\mathrm{Ca}-\mathrm{B}-\mathrm{H}$ phase diagram, variablecomposition structural searches at fixed pressures were carried out using evolutionary algorithms as implemented in the Universal structure predictor: evolutionary Xtallography software (USPEX) [53]. Oversampling of the same minima is avoided through the use of a antiseeding technique. Each structure underwent a five-step relaxation to minimize stress and forces, calculated within DFT. For this purpose we employed the Vienna $a b$ initio software package (VASP) [77], using projector augmented waves (PAW) pseudopotentials with PerdewBurke-Ernzerhof (PBE) exchange-correlation functional supplied within the VASP package. We used a cutoff on the plane wave expansion of $600 \mathrm{eV}$; for reciprocal-space integration, we employed a regular grid in $k$ space with a 0.04 spacing in units of $\frac{2 \pi}{\AA}$, and a gaussian smearing of $0.05 \mathrm{eV}$.

The ternary convex hulls in Fig. 1 were constructed as follows: first, an exploratory run was carried out on the full compositional space, for a total of about 2000 structures per pressure, with a variable number of atoms/cell, ranging from 8 to 16 . This was sufficient to correctly predict some stable stoichiometries at room pressure, but not all of them were identified. To improve the accuracy, we performed additional searches for variable compositions, along selected lines: $\left(\mathrm{Ca}_{1} \mathrm{~B}_{2}\right)_{1-x} \mathrm{H}_{x},\left(\mathrm{Ca}_{2} \mathrm{~B}_{2}\right)_{1-x} \mathrm{H}_{x},\left(\mathrm{Ca}_{2} \mathrm{~B}_{1}\right)_{1-x} \mathrm{H}_{x}$, and $\mathrm{Ca}_{1-x}(\mathrm{BH})_{x}, \mathrm{Ca}_{1-x} \mathrm{~B}_{x}, \mathrm{Ca}_{1-x} \mathrm{H}_{x}$, and $\mathrm{B}_{1-x} \mathrm{H}_{x}$, with about 250 structures per line, and a cell size ranging from 8 to 18 . This was sufficient to reproduce results from Refs. [39,64,65], and represents a good compromise between exploration and sampling. As an example, the convex hull at $0 \mathrm{GPa}$ before refinement including all the compositions sampled in the search is shown in Fig. 7. Finally, we performed a few fixedcomposition structural searches on the compositions which resulted to be stable, to correctly assess the details of the crystal structure. Structures in literature which had a cell size exceeding these parameters were included by hand.

After these exploratory runs, stable or weakly-metastable structures were further relaxed with tighter constraints, until the individual components of the forces were less than $1 \mathrm{meV} / \AA$; finally, the total energy was computed using the tetrahedron method for $\mathrm{k}$-space integration, with a resolution of $0.03 \frac{2 \pi}{\AA}$, resulting in a refinement on the estimate of the energies. From the enthalpies thus calculated, the convex hulls, and the figures, were constructed using the PYMATGEN library [78].

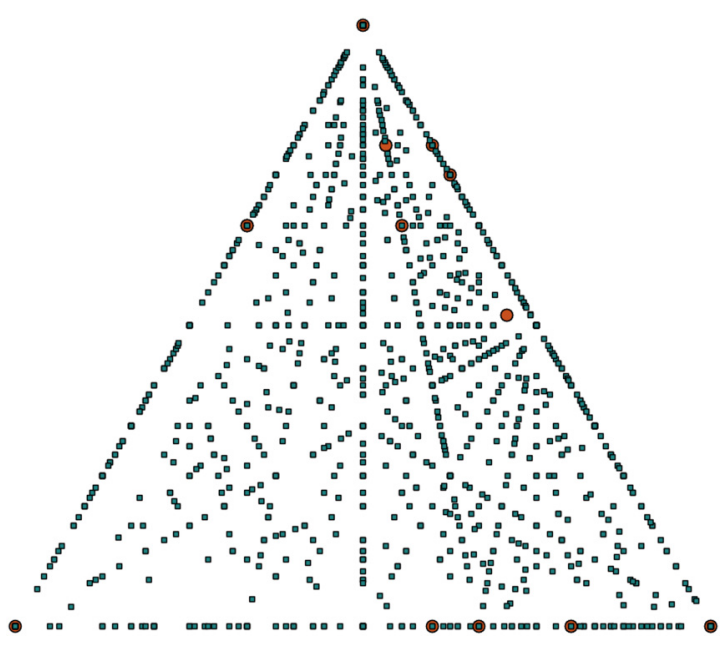

FIG. 7. Convex hull (before refinement) for the $\mathrm{Ca}_{x} \mathrm{~B}_{y} \mathrm{H}_{z}$ system at $0 \mathrm{GPa}$, resulting from exploratory runs over all compositions and over specific lines. A total of about 800 different stoichiometries were sampled with about 4000 structures, amounting to an average of 5 structures/composition, with more structures sampled near minima of the formation enthalpy.

The pressure-dependent phase diagram in Fig. 3 was obtained calculating the energy versus volume relation for each stable and metastable phase identified at 0, 50, 100, and $300 \mathrm{GPa}$; the obtained relation was then fitted using the BirchMurnaghan equation of state. The phase transitions within a single composition were determined by directly comparing the enthalpy thus estimated. The decomposition pressure for a given stoichiometry was estimated comparing the enthalpy of the reactant $r$ with that of the products $i$, according to the formula:

$$
\Delta H=H\left[\mathrm{Ca}_{x_{r}} \mathrm{~B}_{y_{r}} \mathrm{H}_{z_{r}}\right]-\sum_{i=1}^{N} \alpha_{i} H\left[\mathrm{Ca}_{x_{i}} \mathrm{~B}_{y_{i}} \mathrm{H}_{z_{i}}\right],
$$

where $\alpha_{i}$ is the relative fraction of product $i$ in the reaction; whence if $\Delta H$ is negative, the phase is stable, as the enthalpy of the reactant is less than the enthalpy of the products, considering as possible decomposition products the phases stable on the convex hull calculated at the closest pressure. Where $\alpha_{i}$ is the relative fraction of product $i$ in the reaction; whence if $\Delta H$ is negative, the phase is stable, as the enthalpy of the reactant is less than the enthalpy of the products.

\section{Superconductivity}

All electronic structure and superconductivity calculations in Secs. III and IV were carried out with QUANTUM ESPRESSO (QE) using norm-conserving (NC) pseudopotentials, and Perdew-Burke-Ernzerhof (PBE) exchange-correlation functional. A cutoff of 80 Ry was used for the plane-wave expansion of the wave functions. The structures were prerelaxed in $\mathrm{QE}$ until each component of the forces acting on single atoms was less than $2 \mathrm{meV} / \AA$. Calculations of the ground-state charge density were carried out using a 0.06 Ry smearing and a $6 \times 6 \times 6$ grid in reciprocal space for $\mathrm{k}$ space integration. Phonon calculations were performed on a $2 \times 2 \times 2$ reciprocal-space grid, and the integration of the 
electron-phonon matrix element on the Fermi surface was carried out using a $24 \times 24 \times 24$ grid, and a gaussian smearing with a width of $270 \mathrm{meV}$ to describe the zero-width limit of the electronic $\delta$ functions.
The phonon density of states was obtained by performing Fourier interpolation on a $20 \times 20 \times 20$ grid. The figures of the crystal structures were generated using VESTA (Visualization for electronic and structural analysis) [79].
[1] N. W. Ashcroft, Phys. Rev. Lett. 21, 1748 (1968).

[2] N. W. Ashcroft, Phys. Rev. Lett. 92, 187002 (2004).

[3] A. P. Drodzov, M. I. Eremets, I. A. Troyan, V. Ksenofontov, and S. I. Shylin, Nature (London) 525, 73 (2015).

[4] D. Duan, Y. Liu, F. Tian, D. Li, X. Huang, Z. Zhao, H. Yu, B. Liu, W. Tian, and T. Cui, Sci. Rep. 4, 6968 (2015).

[5] A. P. Drodzov, P. Kong, V. Minkov, S. Besedin, M. Kuzovnikov, S. Mozaffari, L. Balicas, F. F. Balakirev, D. E. Graf, V. B. Prakapenka, E. Greenberg, D. A. Knyazev, M. Tkacz, and M. I. Eremets, Nature (London) 569, 528 (2019).

[6] M. Somayazulu, M. Ahart, A. K. Mishra, Z. M. Geballe, M. Baldini, Y. Meng, V. V. Struzhkin, and R. J. Hemley, Phys. Rev. Lett. 122, 027001 (2019).

[7] I. A. Troyan, D. V. Semenok, A. G. Kvashnin, A. V. Sadakov, O. A. Sobolevskiy, V. M. Pudalov, A. G. Ivanova, V. B. Prakapenka, E. Greenberg, A. G. Gavriliuk, V. V. Struzhkin, A. Bergara, I. Errea, R. Bianco, M. Calandra, F. Mauri, L. Monacelli, R. Akashi, and A. R. Oganov, arXiv:1908.01534.

[8] D. V. Semenok, A. G. Kvashnin, A. G. Ivanova, V. Svitlyk, V. Y. Fominski, A. V. Sadakov, O. A. Sobolevskiy, V. M. Pudalov, I. A. Troyan, and A. R. Oganov, Mat. Tod. 33, 36 (2020).

[9] A. P. Drodzov, M. I. Eremets, and I. A. Troyan, arXiv:1508.06224.

[10] P. P. Kong, V. S. Minkov, M. A. Kuzovnikov, S. P. Besedin, A. P. Drodzov, S. Mozaffari, L. Balicas, F. F. Balakirev, V. B. Prakapenka, E. Greenberg, D. A. Knyazev, and M. I. Eremets, arXiv:1909.10482.

[11] A. Oganov, C. Pickard, Q. Zhu, and R. Needs, Nat. Rev. Mater. 4, 331 (2019).

[12] S. Baroni, S. de Gironcoli, and A. D. Corso, Rev. Mod. Phys. 73, 515 (2001).

[13] P. B. Allen and R. C. Dynes, Phys. Rev. B 12, 905 (1975).

[14] L. N. Oliveira, E. K. U. Gross, and W. Kohn, Phys. Rev. Lett. 60, 2430 (1988).

[15] A. Sanna, J. A. Flores-Livas, A. Davydov, K. Dewhurst, S. Sharma, and E. K. U. Gross, J. Phys. Soc. Jpn. 87, 041012 (2018)

[16] J. A. Flores-Livas, L. Boeri, A. Sanna, G. Profeta, R. Arita, and M. Eremet, Phys. Rep. 856, 1 (2020).

[17] J. M. An and W. E. Pickett, Phys. Rev. Lett. 86, 4366 (2001).

[18] L. Boeri, J. Kortus, and O. K. Andersen, Phys. Rev. Lett. 93, 237002 (2004).

[19] D. V. Semenok, I. A. Kruglov, I. A. Savkin, A. G. Kvashnin, and A. R. Oganov, Curr. Opin. Solid State Mater. Sci. 24, 100808 (2020).

[20] H. Wang, X. Li, G. Gao, Y. Li, and Y. Ma, WIREs Comp. Molecul. Sci. 8, e1330 (2018).

[21] E. Zurek and T. Bi, J. Chem. Phys. 150, 050901 (2019).

[22] C. J. Pickard, I. Errea, and M. I. Eremets, Ann. Rev. Condens. Matter Phys. 11, 57 (2020).
[23] M. Benoit, M. Bernasconi, P. Focher, and M. Parrinello, Phys. Rev. Lett. 76, 2934 (1996).

[24] J. A. Flores-Livas, M. Grauzinyte, L. Boeri, G. Profeta, and A. Sanna, Eur. Phys. J. B. 91, 176 (2018).

[25] L. Sun, Z. Zhao, A. L. Ruoff, C.-S. Zha, and G. Stupian, J. Phys. Condens. Matter 19, 425206 (2007).

[26] M. I. Eremets, I. A. Troyan, S. A. Medvedev, J. S. Tse, and Y. Yao, Science 319, 1506 (2008).

[27] J. Feng, W. Grochala, T. Jaron, R. Hoffmann, A. Bergara, and N. W. Ashcroft, Phys. Rev. Lett. 96, 017006 (2006).

[28] C. J. Pickard and R. J. Needs, Phys. Rev. Lett. 97, 045504 (2006).

[29] J. A. Flores-Livas, M. Amsler, C. Heil, A. Sanna, L. Boeri, G. Profeta, C. Wolverton, S. Goedecker, and E. K. U. Gross, Phys. Rev. B 93, 020508(R) (2016).

[30] Y. Li, J. Hao, H. Liu, Y. Li, and Y. Ma, J. Chem. Phys. 140, 174712 (2014).

[31] M. M. Davari Esfahani, A. R. Oganov, H. Niu, and J. Zhang, Phys. Rev. B 95, 134506 (2017).

[32] G. Gao, A. R. Oganov, A. Bergara, M. Martinez-Canales, T. Cui, T. Iitaka, Y. Ma, and G. Zou, Phys. Rev. Lett. 101, 107002 (2008).

[33] H. Zhang, X. Jin, Y. Lv, Q. Zhuang, Q. Lv, Y. Liu, K. Bao, D. Li, B. Liu, and T. Cui, Phys. Chem. Chem. Phys. 17, 27630 (2015).

[34] J. A. Flores-Livas, A. Sanna, and E. K. U. Gross, Eur. Phys. J. B. 89, 63 (2016).

[35] I. Errea, M. Calandra, C. J. Pickard, J. Nelson, R. J. Needs, Y. Li, H. Liu, Y. Zhang, Y. Ma, and F. Mauri, Phys. Rev. Lett. 114, 157004 (2015).

[36] C. Heil and L. Boeri, Phys. Rev. B 92, 060508(R) (2015).

[37] R. Akashi, W. Sano, R. Arita, and S. Tsuneyuki, Phys. Rev. Lett. 117, 075503 (2016).

[38] F. Peng, Y. Sun, C. J. Pickard, R. J. Needs, Q. Wu, and Y. Ma, Phys. Rev. Lett. 119, 107001 (2017).

[39] H. Wang, J. S. Tse, K. Tanaka, T. Iitaka, and Y. Ma, Proc. Natl. Acad. Sci. USA 109, 6463 (2011).

[40] X. Feng, J. Zhang, G. Gao, H. Liu, and H. Wang, RSC Adv. 5, 59292 (2015).

[41] C. Heil, S. di Cataldo, G. B. Bachelet, and L. Boeri, Phys. Rev. B 99, 220502(R) (2019).

[42] H. Liu, I. I. Naumov, R. Hoffmann, N. W. Ashcroft, and R. J. Hemley, Proc. Natl. Acad. Sci. USA 114, 6990 (2017).

[43] The periodic table has 118 elements, therefore there are $\left(\begin{array}{c}118 \\ 2\end{array}\right)=$ 6903 possible combinations.

[44] D. Li, Y. Liu, F. Tian, S.-L. Wei, Z. Liu, D.-F. Duan, B. Liu, and T. Cui, Front. Phys. 13, 137107 (2018).

[45] W. Cui, T. Bi, J. Shi, Y. Li, H. Liu, E. Zurek, and R. J. Hemley, Phys. Rev. B 101, 134504 (2020).

[46] Y. Ge, F. Zhang, and Y. Yao, Phys. Rev. B 93, 224513 (2016). 
[47] B. Liu, W. Cui, J. Shi, L. Zhu, J. Chen, S. Lin, R. Su, J. Ma, K. Yang, M. Xu, J. Hao, A. P. Durajski, J. Qi, Y. Li, and Y. Li, Phys. Rev. B 98, 174101 (2018).

[48] M. Amsler, Phys. Rev. B 99, 060102(R) (2019).

[49] X. Liang, S. Zhao, C. Shao, A. Bergara, H. Liu, L. Wang, R. Sun, Y. Zhang, Y. Gao, Z. Zhao, X.-F. Zhou, J. He, D. Yu, G. Gao, and Y. Tian, Phys. Rev. B 100, 184502 (2019).

[50] X. Liang, A. Bergara, L. Wang, B. Wen, Z. Zhao, X.-F. Zhou, J. He, G. Gao, and Y. Tian, Phys. Rev. B 99, 100505(R) (2019).

[51] C. Kokail, W. von der Linden, and L. Boeri, Phys. Rev. Mater. 1, 074803 (2017).

[52] Y. Sun, J. Lv, Y. Xie, H. Liu, and Y. Ma, Phys. Rev. Lett. 123, 097001 (2019).

[53] C. W. Glass, A. R. Oganov, and N. Hansen, Comp. Phys. Commun. 175, 713 (2006).

[54] P. Giannozzi, S. Baroni, N. Bonini, M. Calandra, R. Car, C. Cavazzoni, D. Ceresoli, G. L. Chiarotti, M. Cococcioni, and I. Dabo, J. Phys. Condens. Matter 21, 395502 (2009).

[55] A. Züttel, A. Borgschulte, and S.-I. Orimo, Scripta Materialia 56, 823 (2007).

[56] M. Paskevicius, L. H. Jepsen, P. Schouwink, R. Cerny, D. B. Ravnsbaek, Y. Filinchuk, M. Dornheim, F. Besenbacher, and T. R. Jensen, Chem. Soc. Rev. 46, 1565 (2017).

[57] Y. Zhang, E. Majzoub, V. Ozolins, and C. Wolverton, Phys. Rev. B 82, 174107 (2010).

[58] See Supplemental Material at http://link.aps.org/supplemental/ 10.1103/PhysRevB.102.014516 for more details on the electronic structure, and crystallographic information files (.cif) for all structures.

[59] M. D. Riktor, M. H. Sørby, K. Chlopek, M. Fichtner, and B. C. Hauback, J. Mater. Chem. 19, 2754 (2009).

[60] Y. Yan, A. Remhof, D. Rentsch, A. Züttel, S. Giri, and P. Jena, Chem. Commun. 51, 11008 (2015).

[61] Y. Kim, S.-J. Hwang, J.-H. Shim, Y.-S. Lee, H. N. Han, and Y. W. Cho, J. Phys. Chem. C 116, 4330 (2012).

[62] Y. Kim, S.-J. Hwang, Y.-S. Lee, J.-Y. Suh, H. N. Han, and Y. W. Cho, J. Phys. Chem. C 116, 25715 (2012).
[63] V. Ozolins, E. H. Majzoub, and C. Wolverton, J. Am. Chem. Soc. 131, 230 (2008).

[64] S. Shah and A. N. Kolmogorov, Phys. Rev. B 88, 014107 (2013).

[65] C.-H. Hu, A. R. Oganov, Q. Zhu, G.-R. Qian, G. Frapper, A. O. Lyakhov, and H.-Y. Zhou, Phys. Rev. Lett. 110, 165504 (2013).

[66] P. C. Aeberhard, K. Refson, P. P. Edwards, and W. I. F. David, Phys. Rev. B 83, 174102 (2011).

[67] X. Li, Y. Huang, S. Wei, D. Zhou, Y. Wang, X. Wang, F. Li, Q. Zhou, B. Liu, X. Huang, and T. Cui, J. Phys. Chem. C 122, 14272 (2018).

[68] V. Stabila, J.-H. Her, W. Zhou, S.-J. Hwang, C. Kim, L. A. M. Ottley, and T. J. Udovic, J. Solid State Chem. 183, 1133 (2010).

[69] V. Natoli, R. M. Marting, and D. M. Ceperley, Phys. Rev. Lett. 70, 1952 (1993).

[70] J. Heyd, G. E. Scuseria, and M. Ernzerhof, J. Chem. Phys., 118, 8207 (2003).

[71] S. Y. Savrasov and D. Y. Savrasov, Phys. Rev. B 54, 16487 (1996).

[72] J. P. Carbotte, Rev. Mod. Phys. 62, 1027 (1990).

[73] I. Errea, F. Belli, L. Monacelli, A. Sanna, T. Koretsune, T. Tadano, R. Bianco, M. Calandra, R. Arita, F. Mauri, and J. A. Flores-Livas, Nature (London) 578, 66 (2020).

[74] W. L. McMillan, Phys. Rev. 167, 331 (1968).

[75] C. Heil, G. B. Bachelet, and L. Boeri, Phys. Rev. B 97, 214510 (2018).

[76] A. D. Grockowiak, M. Ahart, T. Helm, W. A. Coniglio, R. Kumar, M. Somayazulu, Y. Meng, M. Oliff, V. Williams, N. W. Ashcroft, R. J. Hemley, and S. W. Tozer, arXiv:2006.03004.

[77] G. Kresse and J. Furthmüller, Phys. Rev. B 54, 11169 (1996).

[78] Ong, S. Ping, W. D. Richards, A. Jain, G. Hautier, M. Kocher, S. Cholia, D. Gunter, V. L. Chevrier, K. A. Persson, and G. Ceder, Comput. Mater. Sci. 68, 314 (2013).

[79] K. Momma and F. Izumi, J. Appl. Crystallogr. 44, 1272 (2011). 